\title{
Unsupervised Pathological Area Extraction Using 3D T2 and FLAIR MR Images
}

\author{
Pavel Dvořák ${ }^{1,2}$, Karel Bartušek $^{2}$ and Zdeněk Smékal ${ }^{1}$ \\ ${ }^{1}$ Deptartment of Telecommunications, Faculty of Electrical Engineering and Communication, Brno University of \\ Technology, Technicka 12, 61200 Brno, Czech Republic, pavel.dvorak@phd.feec.vutbr.cz \\ ${ }^{2}$ ASCR, Institute of Scientific Instruments, Kralovopolska 147, 61264 Brno, Czech Republic
}

\begin{abstract}
This work discusses fully automated extraction of brain tumor and edema in 3D MR volumes. The goal of this work is the extraction of the whole pathological area using such an algorithm that does not require a human intervention. For the good visibility of these kinds of tissues both T2-weighted and FLAIR images were used. The proposed method was tested on 80 MR volumes of publicly available BRATS database, which contains high and low grade gliomas, both real and simulated. The performance was evaluated by the Dice coefficient, where the results were differentiated between high and low grade and real and simulated gliomas. The method reached promising results for all of the combinations of images: real high grade $(0.73 \pm 0.20)$, real low grade $(0.81 \pm$ 0.06), simulated high grade $(0.81 \pm 0.14)$, and simulated low grade $(0.81 \pm 0.04)$.
\end{abstract}

Keywords: Brain tumor, brain tumor segmentation, extraction, magnetic resonance, MRI, pathology, segmentation, symmetry analysis.

\section{INTRODUCTION}

$\mathrm{S}$ NCE THE MR technique is becoming more popular due to its non-invasive principle, the imaging of biological structures by MR equipments is a routine investigating procedure today [1]. For this reason, the automatic processing of this kind of images is getting more attention. Nowadays, the issue of automatic analysis of brain tumors is of great interest. It is the first step in surgical and therapy planning. And the very first step of the automatic analysis of brain tumor is its detection and subsequent segmentation. The difficulty of the tumor segmentation is in its shape variability in each case.

So far, the proposed general brain MR segmentation algorithms, with a few exceptions (such as [2], which mention it peripherally), do not deal with this task at all. Hence, concrete algorithms have to be developed.

The automatic segmentation of brain tumors is still a challenging problem, even though several different and interesting fully- or semi-automatic algorithms have been proposed in recent years. The existing algorithms can be classified into semi- and fully-automatic methods from a user viewpoint and into region- and contour-based methods from technical viewpoint.

The semi-automatic [3], [4] require some user interaction, e.g., to select the starting point lying inside the tumor or to select several points of foreground and several points of background. The automatic [5], [6] methods do not require any interaction and are usually based on prior knowledge of the human brain structure, either tissue atlas or left-right symmetry, or their combination.

The region-based methods [7], [8] usually search for pixels or regions with similar properties that create connected regions. This includes, e.g., region growing, classification, etc. The contour-based methods [9] use image gradient in particular area to detect a boundary and create a region for subsequent boundary evolution. The most famous method of this group is active contours [10]. Some works [11], [12] use combination of region-based and contour-based methods and try to combine the advantages of both of these groups.

At present, multi-parametric image analysis is frequently discussed within the scientific community [13]. This technique, even though it can be based on traditional segmentation methods (thresholding, active contours), exploits information obtained from more images or modalities at the same time.

This is widely used in algorithms using machine learning and classification approach. Varieties of these machine learning methods can be found in the proceedings of the MICCAI 2012 Challenge on Multimodal Brain Tumor Segmentation (http://www.imm.dtu.dk/projects/BRATS2012). This challenge and the obtained results showed that the current methods still do not reach the sufficient accuracy. This publicly available database has reached high interest and is widely used for evaluation of current algorithms, e.g., in [14], [15], where the later one deals only with segmentation of the core of low grade gliomas and reached promising results.

However, these methods still have limitations. The main one is the transferability to the MR volumes acquired with different parameters. For this purpose, the intensity normalization has to be used. Many methods have been developed for this task as well, but even a small shift can cause the failure of classification algorithms, which in almost all cases relies on the image intensities. This was shown in [14], where promising results were achieved, when the model was trained on the same database, while after testing on a different database, the performance dropped significantly.

The current methods usually rely also on T1-weighted contrast enhanced images [16]. This is the image we are trying to avoid, since it requires a contrast enhanced agent (usu- 
ally gadolinium) to be injected into the patient's blood, which breaks the non-invasivity of magnetic resonance.

The general properties of healthy brain are widely used as a prior-knowledge. One of them is the probability of tissues locations using probabilistic brain atlas, which requires manual labeling of template MRI and is used, e.g., in [17]. Another widely used knowledge, which is used in this article, is the approximate left-right symmetry of healthy brain. This approach is also used, e.g., in [5], [6]. The areas breaking this symmetry are most likely parts of a tumor or an edema.

Consequently, in this work a fully automated method based on symmetry analysis is proposed. Using the symmetry analysis, the preliminary locating of this kind of pathological area is found, followed by the border delimitation, which is performed by Otsu's thresholding technique [18] in both image modalities using the knowledge of the brain tumor manifestation in both of them. The details of the proposed algorithm are described in section 2 .

The rest of the paper is organized as follows. In section 3 , the data used for evaluation and the evaluation criteria are described. Section 4 describes the performance of the algorithm and its comparison with other state-of-the-art methods. The conclusion of the paper is given in section 5 .

\section{PRoposed Method}

The input of the whole process is registered 3D T2 and FLAIR magnetic resonance volumes. The reason for these contrast volumes is the good visibility of this kind of pathology in both of them. However, it is not always possible to separate it from healthy tissue using only one of these contrasts.

The flow diagram of the proposed method is depicted in Fig. 1. The input of the whole process is a 3D magnetic resonance volume containing a tumor. The algorithm does not deal with the tumor presence detection, but this topic, unfortunately dealing only with 2D images, can be found in our previous work [19]. The pathology extraction process consists of three steps described in detail in subsequent subsections. These steps are preprocessing, symmetry analysis, and tumor extraction.

\subsection{Preprocessing}

The first step is the extraction of brain, followed by cutting the volume. Since all images in testing BRATS database contain only brain, this step was skipped during testing. However, there are several methods used for brain extraction from 3D volumes such as [20]. This algorithm has been created and implemented by our colleagues and can be easily used. Subsequently, the regions outside the brain volume are cut off to ensure that only relevant areas are analyzed. From this new cut volume, the mid-sagittal plane has to be extracted to correctly align the head. Several methods exist for this task as well, e.g., the one based on bilateral symmetry maximization [21] can be used.

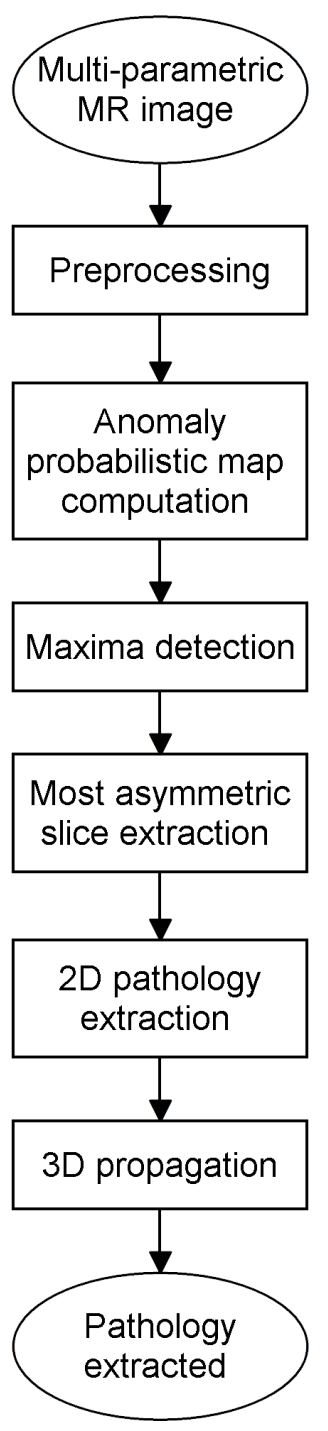

Fig. 1: The flow diagram of the proposed method.

\subsection{Symmetry analysis}

The first part of this work is the detection of symmetry anomalies, which are usually caused by a brain tumor, whose detection is the main purpose of this article. The first step of this process is dividing of the input volume into left and right halves.

Assuming that the head is not rotated and the skull is approximately symmetric, the symmetry axis is parallel to the sagittal plane and divides the volume of the brain into two parts of the same size. Since the method is not pixel-based, the precision of the determined symmetry axis has not significant influence.

A cubic block, with the side length computed as one quarter of the cropped image side length, is created. This size and sizes computed in the following computation are suitable for the detection of both small and large tumors. The algorithm goes through both halves symmetrically by this block. The step size is smaller than the block size to ensure the overlapping of particular areas. These areas are compared with their 

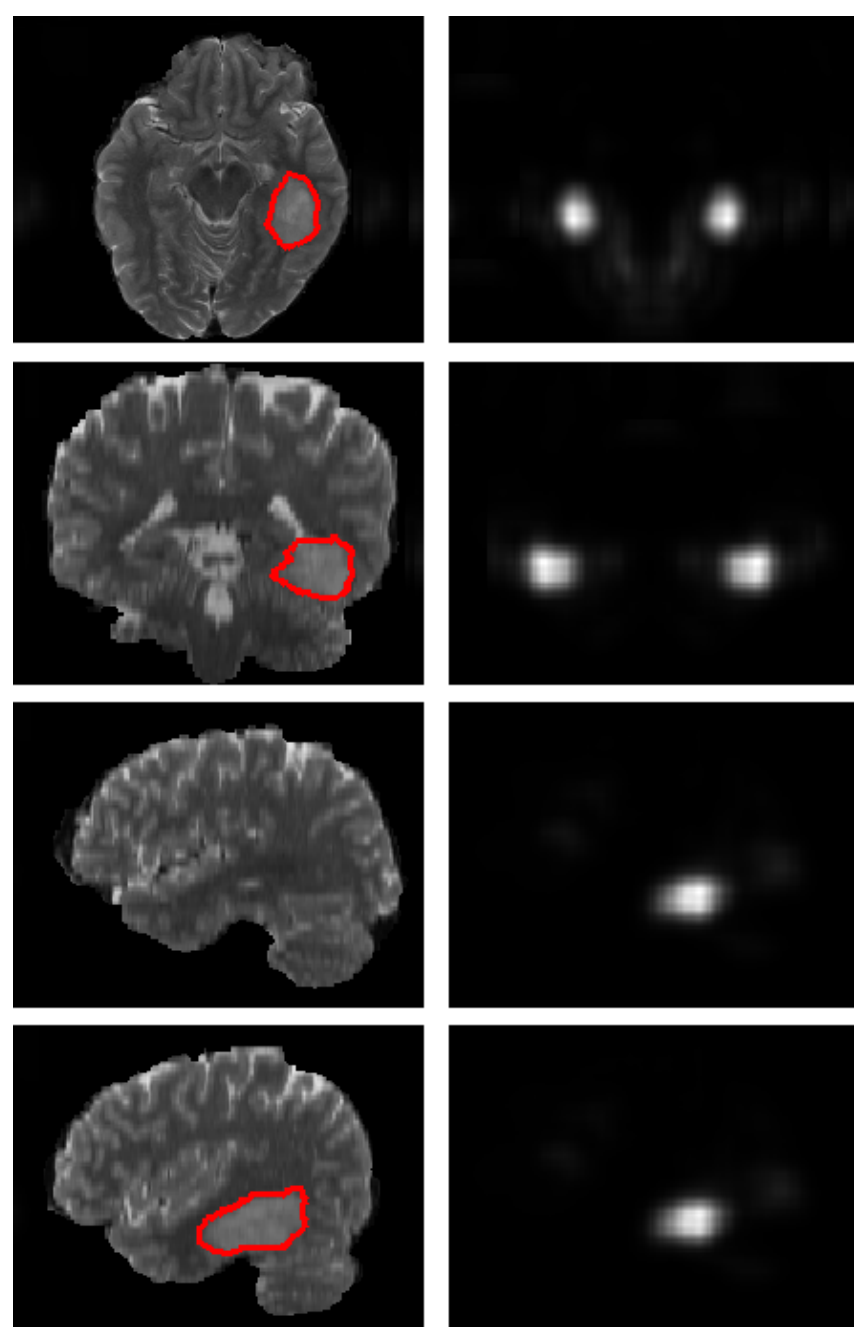

Fig. 2: Example of the brain tumor probability map on volumes from real data of the low grade glioma. The left column shows the slices detected with the highest Asymmetry Coefficient. The right column shows the asymmetry maps of the T2-weighted volume of the corresponding slices.

opposite symmetric parts.

The comparison is done by the Bhattacharyya Coefficient [22], which was also used previously in [6]. Normalized histograms with the same range are computed from both parts and the Bhattacharyya Coefficient (BC) is computed from these histograms as follows [22]:

$$
B C=\sum_{i=1}^{N} \sqrt{l(i) \cdot r(i)}
$$

where $N$ denotes the number of bins in the histogram, $l$ and $r$ denote histograms of blocks in left and right half, respectively.

The range of values of $\mathrm{BC}$ is $\langle 0,1\rangle$, where the smaller the value, the bigger the difference between histograms. For the next computation, the Asymmetry Coefficient is computed as $A C=1-B C$.

The Asymmetry Coefficient (AC) is computed for all blocks. Since the step size is smaller than the block size, the overlap exists and more values of $\mathrm{AC}$ are present for most pix- els. To obtain the appropriate asymmetry map, the mean of all values computed for a particular pixel is computed.

The computed ACs create the asymmetry map, where the higher value expresses the higher probability of tumor presence in particular location. The example of such asymmetry map is depicted in Fig. 2.

\subsection{Multi-resolution probabilistic map computation}

The whole cycle of symmetry checking is repeated four times, but with different size of block. Height and width of the block are iteratively reduced to the half of the previous value. So the size of the block is $1 / 1,1 / 4,1 / 16$, and $1 / 64$ of the initial size, respectively. The purpose of smaller areas is the more precise detection of asymmetry. This approach corresponds to multiresolution image analysis described in [23]. A block size of $1 / 256$ of the initial size was tested as well, but the results were not improved and the maximum of AC for this block size was equal to 1 for every image in database.

The output of each cycle is a probabilistic map of anomalies. The product of values corresponding to a particular pixel is computed. The output is the new multi-resolution probabilistic map.

\subsection{Pathology extraction}

The extraction process starts in the axial slice where the highest AC was detected and it is then propagated into the whole $3 \mathrm{D}$ volume. The method using the pathology extraction directly from the 3D volume was tested as well. This was faster, but the performance was poorer.

\subsubsection{Extraction from the most asymmetric slice}

For the pathology extraction purpose, the thresholding of the multi-resolution asymmetry map is performed by the value of $10 \%$ of the maximal asymmetry. This value was set experimentally and it ensures that at least a small region is extracted. The result is the both-sided mask that contains both the tumor on one side and the healthy tissue on the other side.

Since multifocal tumor can appear, the detection process is not limited to only one region. All regions created by thresholding are considered. As a result, multifocal tumors located in both halves asymmetrically can be correctly detected.

The subsequent extraction process is based on the method proposed in [24]. The glioma and the edema can be well separated using T2-weighted volume, since they appear hyperintense in this MR contrast. The automatic thresholding can be performed to extract these pathological areas.

The threshold is determined using Otsu's method [18] from the points inside the resulting mask of asymmetry detection, but the thresholding process is applied to the whole image. According to the algorithm proposed by Otsu in 1979, the optimal threshold $k^{*}$ is such threshold with the following property:

$$
\sigma_{B}^{2}\left(k^{*}\right)=\max _{1 \leq k \leq L} \sigma_{B}^{2}(k),
$$



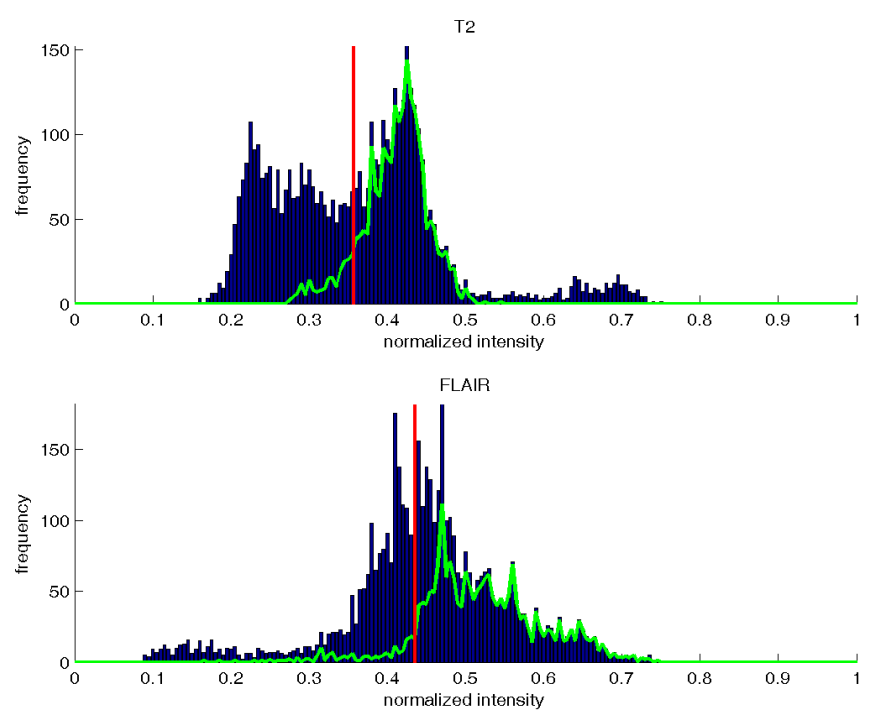

Fig. 3: Example of threshold determination using Otsu's algorithm for T2-weighted and FLAIR image of high grade glioma. Blue: histogram of the whole asymmetric area, Green: histogram of true pathology, Red: computed threshold.

where $L$ is the number of intensity values in region and $\sigma_{B}^{2}(k)$ denotes the between-class variance for threshold $k$, it is based on class means and is computed according to the equation:

$$
\sigma_{B}^{2}(k)=\omega_{0} \omega_{1}\left(\mu_{0}-\mu_{1}\right)^{2},
$$

where $\omega_{0}, \omega_{1}$ mean the probabilities of class occurrence and $\mu_{0}, \mu_{1}$ mean the class mean levels for classes 0 and 1 , respectively.

Two thresholds are computed by Otsu's method, one for each image (T2 and FLAIR). The computed thresholds are the global thresholds used in propagation as well.

An example of the computed threshold from asymmetric region in T2-weighted and FLAIR image of the same slice is depicted in Fig. 3, where histograms of the whole asymmetric area (blue bar graph), the true pathological area (green curve), and the computed thresholds (red line) are shown. The combination of both images and their thresholding is shown by scatter graph in Fig. 4. As it can be seen in both these images, the pathology could not be separated in neither of these images, but combination of both brings improvement.

Morphological erosion and dilation are performed with the resulting mask to smooth the region borders and separate regions connected by a thin area. The conjunction of these masks is found. Since some incorrect areas could be extracted, only those which are situated mostly inside the asymmetry region are labeled as pathological. Regions with the size smaller than $10 \%$ of the largest segment are eliminated as well. Since the pathological area could extend beyond the asymmetry area border, the whole region created by the thresholding is extracted.

The resulting mask $M$ of the extraction process is created as $M=M_{T 2} \bigcap M_{F L A I R}$, where $M_{T 2}$ and $M_{F L A I R}$ denote the thresholding mask of T2-weighted and FLAIR image, respectively.

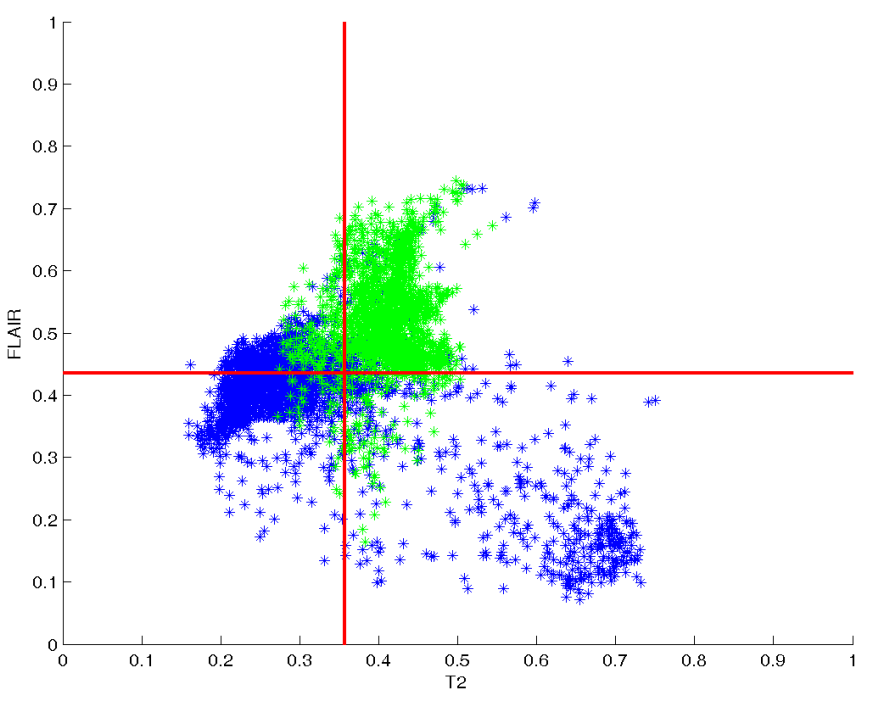

Fig. 4: Example of combination of T2 and FLAIR images for pathology extraction using thresholding of the high grade glioma used in Fig. 3. Blue: values of the whole asymmetric area, Green: values of true pathology, Red: computed thresholds for both images. Values of pixels labeled as pathological are situated above both thresholds, i.e. in top right rectangle.

This corresponds to the pixels with intensities situated in top right rectangle in Fig. 4.

\subsubsection{Propagation into neighbor slices}

Once the pathology is extracted from the most asymmetric axial slice, it can be propagated into other slices. At first both 3D volumes are thresholded using the particular threshold value computed in the previous step. In order to avoid extraction of healthy areas far from the pathological ones, the propagation of neighbor mask is necessary.

Propagation starts with both neighbor axial slices and then continues in both directions. The result for $n$-th slice can be defined as:

$$
M(n)=M_{T 2}(n) \bigcap M_{F L A I R}(n) \bigcap M_{D}(n \pm 1),
$$

where $M_{D}(n \pm 1)$ means the dilated mask from neighbor slice and its sign depends on the direction of propagation. The dilation is necessary in order to include shifted or enlarged regions.

\section{TESTING}

\subsection{Test dataset}

Brain tumor image data used in this work were obtained from the MICCAI 2012 Challenge on Multimodal Brain Tumor Segmentation (http://www.imm.dtu.dk/ projects/BRATS2012) organized by B. Menze, A. Jakab, S. Bauer, M. Reyes, M. Prastawa, and K. Van Leemput. The challenge database contains fully anonymized images from the following institutions: ETH Zurich, University of Bern, University of Debrecen, and University of Utah. 
Table 1: Detection performance in particular sets

For each patient, T1, T2, FLAIR, and post-Gadolinium T1 MR volumes are available. All volumes are linearly coregistered to the T1 contrast image, skull stripped, and interpolated to $1 \mathrm{~mm}$ isotropic resolution. No attempt was made to put the individual patients in common reference space. The data contains real volumes of 20 high-grade and 10 low-grade glioma subjects and simulated volumes of 25 high-grade and 25 low-grade glioma subjects. All the simulated images are in BrainWeb space. [25] [26]

\subsection{Evaluation criteria}

The suitability of all available contrast volumes was tested for detection purpose by the proposed algorithm, as well as the combination of all of them. As a combination, the average asymmetry and the product of all asymmetries was used.

The evaluation criterion was the position of the maximum AC compared to ground truth. Since there were two maxima of the asymmetry map, one for each half, either of them was considered.

The extraction process was evaluated by the Dice Coefficient (DC) [27], which is computed according to the equation:

$$
D C=\frac{2|A \bigcap B|}{|A|+|B|},
$$

where $A$ and $B$ denote the ground truth and the result masks of the extraction, respectively. The DC compares the intersection of two sets with their union. The range of the DC values is $\langle 0 ; 1\rangle$, where 1 expresses the perfect segmentation.

Another measure widely employed for segmentation evaluation is Accuracy (A), used in, e.g., [3] and defined by the equation:

$$
A=\frac{T P+T N}{T P+F P+T N+F N},
$$

where $T P, F P, F N$ and $T N$ stand for "True Positive", "False Positive", "False Negative", and "True Negative", respectively. This measure is in the same range as DC and the higher value indicates the better performance as well.

\section{RESULTS}

In this section, the performance of both parts of the algorithm is described and it is within the bounds of possibility compared with other methods tested on the same database. At first, the locating accuracy is evaluated followed by the evaluation of the extraction process.

\subsection{Pathology location}

The results for particular sets of BRATS database and for particular contrast volumes are shown in Table 1.

It has to be stated that these results are for non-aligned volumes. The method would not work for highly rotated volumes, but, since it is region-based rather than pixel-based, the perfect alignment was not necessary. From Table 1, it can be seen that the most suitable for anomaly detection are T2weighted and FLAIR MR contrasts.

\begin{tabular}{|l||r|r|r|r|}
\hline & T1 & T1 - CE & \multicolumn{1}{c|}{ T2 } & FLAIR \\
\hline Real HG & $60 \%$ & $70 \%$ & $85 \%$ & $90 \%$ \\
Real LG & $60 \%$ & $90 \%$ & $100 \%$ & $100 \%$ \\
\hline Real & $60 \%$ & $77 \%$ & $90 \%$ & $93 \%$ \\
\hline Sim. HG & $100 \%$ & $100 \%$ & $100 \%$ & $96 \%$ \\
Sim. LG & $100 \%$ & $100 \%$ & $100 \%$ & $100 \%$ \\
\hline Sim. & $100 \%$ & $100 \%$ & $100 \%$ & $98 \%$ \\
\hline \hline Overall & $85 \%$ & $91 \%$ & $96 \%$ & $96 \%$ \\
\hline
\end{tabular}

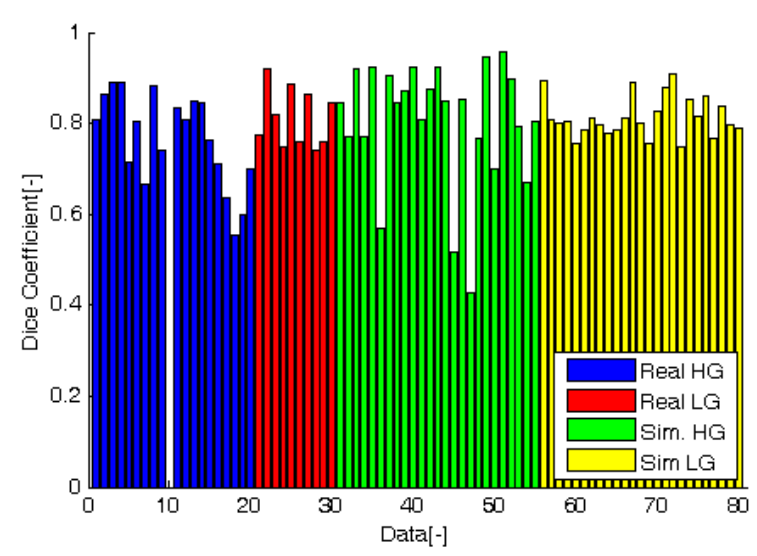

Fig. 5: The Dice Coefficient for particular volumes in database.

\subsection{Pathology extraction}

The Dice Coefficient for particular datasets is shown in Fig. 5. The overall results of extraction process evaluated by mean and median Dice Coefficient and Accuracy are summarized in Table 2. Mean \pm std and Median \pm mad were computed for both coefficients.

Even though the maximum of the FLAIR and T2 asymmetry map was situated outside the ground truth in 3 of 80 cases, there was no intersection between ground truth and automatic extraction result only in one of them. In other words, even though the maximum was located outside the tumor, the extracted regions contained this pathological area.

For the real high grade gliomas, the results correspond to the average from all cases. If the case, where there was no intersection and the Dice Coefficient was equal to zero, was omitted, the average DC would be equal to $0.77 \pm 0.10$ and the real data DC would be equal to $0.79 \pm 0.09$, the overall DC would remain the same. In this case, the accuracy would be equal to $0.96 \pm 0.03,0.97 \pm 0.03$, and $0.97 \pm 0.02$ for real high grade glioma, real glioma, and overall database, respectively.

The examples of extraction results on the real data of the low grade glioma and the simulated data of the high grade multifocal glioma are shown in Fig. 6 and 7, respectively. In both figures, the left column shows the slices detected with the highest probability of the pathological area presence (with maximal value of the asymmetry map computed from T2weighted volume), where the red color depicts the ground truth and the blue color shows the result of tumor extrac- 
MEASUREMENT SCIENCE REVIEW, Volume 14, No. 6, 2014

Table 2: Extraction performance in particular sets

\begin{tabular}{|l||c|c|c|c|}
\hline \multicolumn{1}{|c||}{} & \multicolumn{2}{c|}{ DC } & \multicolumn{2}{c|}{$\mathrm{A}$} \\
\hline & mean & median & mean & median \\
\hline Real HG & $0.73 \pm 0.20$ & $0.78 \pm 0.09$ & $0.96 \pm 0.03$ & $0.97 \pm 0.02$ \\
Real LG & $0.82 \pm 0.06$ & $0.81 \pm 0.05$ & $0.98 \pm 0.01$ & $0.99 \pm 0.00$ \\
\hline Real & $0.76 \pm 0.17$ & $0.80 \pm 0.07$ & $0.97 \pm 0.03$ & $0.97 \pm 0.02$ \\
\hline Sim. HG & $0.81 \pm 0.14$ & $0.85 \pm 0.06$ & $0.97 \pm 0.02$ & $0.97 \pm 0.02$ \\
Sim. LG & $0.78 \pm 0.04$ & $0.77 \pm 0.03$ & $0.97 \pm 0.01$ & $0.97 \pm 0.01$ \\
\hline Sim. & $0.79 \pm 0.05$ & $0.78 \pm 0.06$ & $0.97 \pm 0.02$ & $0.97 \pm 0.01$ \\
\hline \hline Overall & $0.78 \pm 0.13$ & $0.79 \pm 0.06$ & $0.97 \pm 0.03$ & $0.97 \pm 0.01$ \\
\hline
\end{tabular}
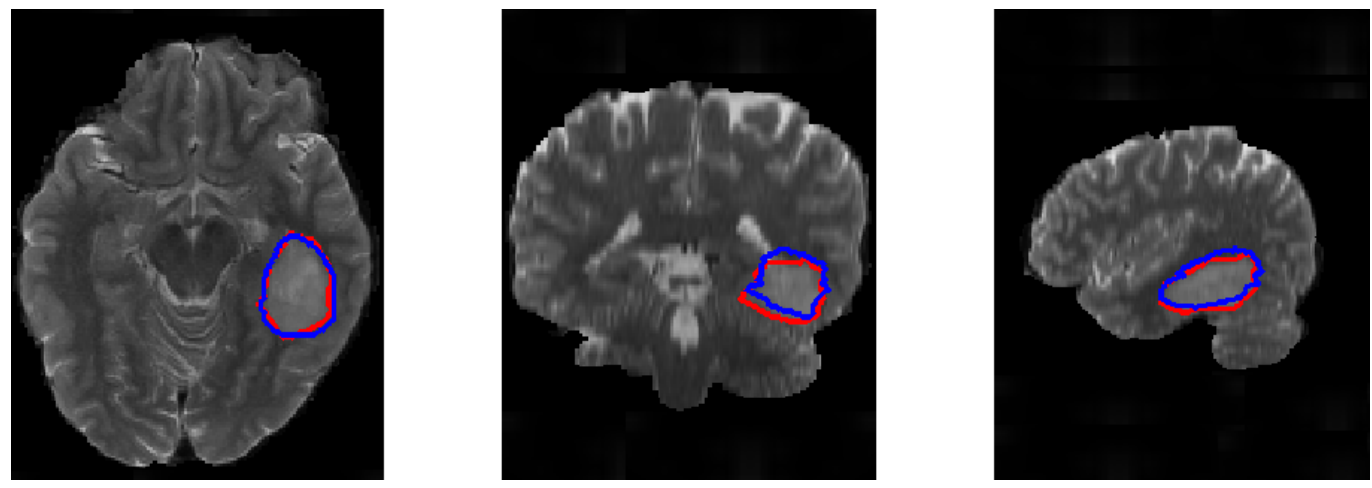

Fig. 6: Example of brain tumor extraction on volumes from real data of the low grade glioma with DC $=0.76$. The particular slices detected with the highest probability of the pathological area presence are shown. The red color depicts the ground truth and the blue color shows the result of tumor extraction using the proposed algorithm.
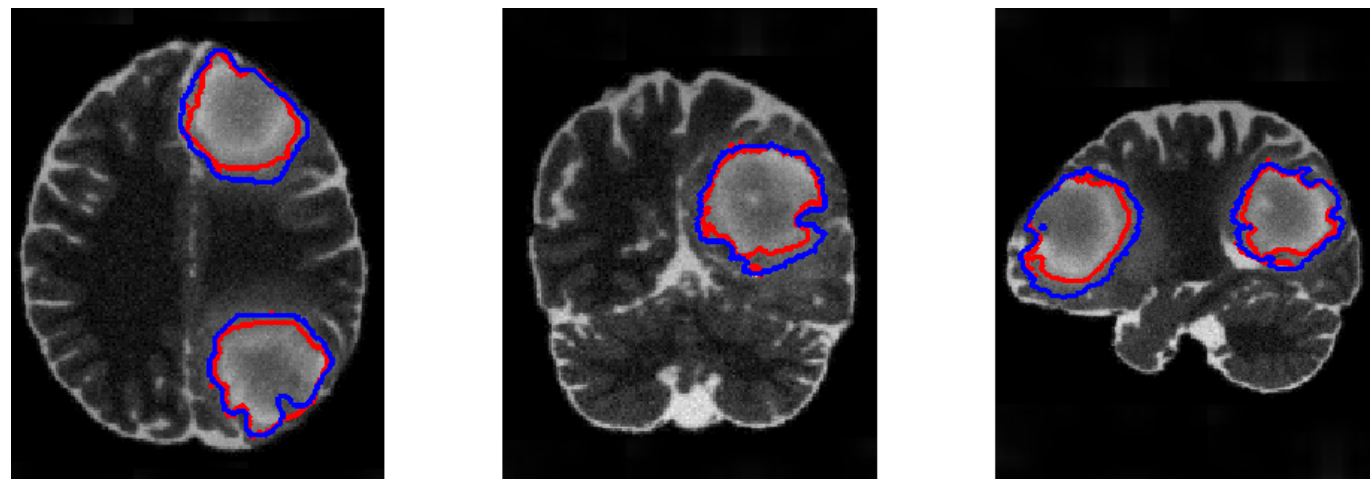

Fig. 7: Example of pathology extraction on volumes from simulated data of multifocal high grade glioma with DC $=0.82$. The particular slices detected with the highest probability of the pathological area presence are shown. The red color depicts the ground truth and the blue color shows the result of tumor extraction using the proposed algorithm.

tion using the proposed method. The right column shows the asymmetry maps computed from T2-weighted volume of the corresponding slices. As can be seen from Fig. 6, the precise vertical alignment of the head was not necessary.

\subsection{Comparison with other methods}

Even though the proposed method was tested on publicly available BRATS 2012 database, the comparison with other methods was not straightforward. The MICCAI 2012 Challenge focused on segmentation of tumor and edema separately. Hence, the described results in this paper could not be compared to those described in the proceedings of MICCAIBRATS 2012 [28]. On the other hand, our work is fully automated and does not require a training phase as all methods proposed in the proceedings. Training phase requires normalized intensities in all involved images, which brings another inaccuracy into the segmentation process and cannot be always reached accurately. An alternative to intensity normalization is a patient specific training dataset that requires manual selection of several points in foreground and background tissues. 


\section{DISCUSSION / CONCLUSION}

The aim of this work was to propose a fully automated method for the extraction of pathological area in 3D brain MR volumes. The proposed method is based on simple image processing tasks, but it reached similar results as the sophisticated machine algorithms, as it was shown in section 4. However, our method significantly outperformed others concerning the runtime with average 33 and 44 seconds per patient for real and simulated data, respectively. Moreover, the transferability and usability on any database is guaranteed by its independence of the image intensities. The algorithm does not contain any training stage and it is based on human detection model. The method showed promising results that could help in automation of the brain tumor segmentation task.

In comparison with other proposed and tested methods, it requires only two modalities, which makes the acquisition and image preprocessing less demanding. Moreover, modalities using contrast agent fluid are not employed here.

The proposed algorithm was able to detect and extract multifocal tumors as separated regions as well, as it was shown in section 4.

However, there are still limitations that will be explored in future work. One of them is the assumption of the tumor presence in the volume. This will be considered and the tumor presence will be detected based on our previous work [19]. The future work will mainly emphasize the segmentation of particular parts of the pathology.

\section{ACKNOWLEDGEMENT}

This research work is funded by project SIX CZ.1.05/2.1.00/03.0072, project COST CZ LD14091, and project GACR 102/12/1104.

\section{REFERENCES}

[1] Gogola, D., Krafcik, A., Strbak, O., Frollo, I. (2013). Magnetic resonance imaging of surgical implants made from weak magnetic materials. Measurement Science Review 13(4), 165-168.

[2] Ahlgren, A., Wirestam, R., Stahlberg, F., Knutsson, L. (2014). Automatic brain segmentation using fractional signal modeling of a multiple flip angle, spoiled gradient-recalled echo acquisition. Magnetic Resonance Materials in Physics, Biology and Medicine.

[3] Mikulka, J., Gescheidtova, E. (2013). An improved segmentation of brain tumor, edema and necrosis. In: Progress in Electromagnetics Research Symposium. pp. 25-28.

[4] Wu, Y., Yang, W., Jiang, J., Li, S., Feng, Q., Chen, W. (2013). Semi-automatic segmentation of brain tumors using population and individual information. Journal of Digital Imaging 26(4), 786-796.

[5] Pedoia, V., Binaghi, E., Balbi, S., De Benedictis, A., Monti, E., Minotto, R. (2012). Glial brain tumor detec- tion by using symmetry analysis. In: Proc. SPIE, Vol. 8314. pp. 831445-831445-8.

[6] Saha, B. N., Ray, N., Greiner, R., Murtha, A., Zhang, H. (2012). Quick detection of brain tumors and edemas: A bounding box method using symmetry. Computerized Medical Imaging and Graphics 36(2), 95-107.

[7] Zhang, N., Ruan, S., Lebonvallet, S., Liao, Q., Zhu, Y. (2011). Kernel feature selection to fuse multi-spectral MRI images for brain tumor segmentation. Computer Vision and Image Understanding 115(2), 256-269.

[8] Corso, J. J., Sharon, E., Yuille, A. (2006). A.: Multilevel segmentation and integrated bayesian model classification with an application to brain tumor segmentation. In: Medical Image Computing and Computer Assisted Intervention. pp. 790-798.

[9] Ho, S., Bullitt, E., Gerig, G. (2002). Level-set evolution with region competition: Automatic 3-D segmentation of brain tumors. In: Proceedings of the 16 th International Conference on Pattern Recognition (ICPR'02) Volume 1. Washington, DC, USA, 10532.

[10] Mikulka, J., Gescheidtova, E., Bartusek, K. (2012). Soft-tissues image processing: Comparison of traditional segmentation methods with $2 \mathrm{D}$ active contour methods. Measurement Science Review 12(4), 153-161.

[11] Cap, M., Gescheidtova, E., Marcon, P., Bartusek, K. (2013). Automatic detection and segmentation of the tumor tissue. In: Progress in Electromagnetics Research Symposium. pp. 53-56.

[12] A.Rajendran, Dhanasekaran, R. (2012). Fuzzy clustering and deformable model for tumor segmentation on MRI brain image: A combined approach. In: Procedia Engineering, International Conference on Communication Technology and System Design 2011, 30, pp. 327 333.

[13] Benes, R., Karasek, J., Burget, R., Riha, K. (2013). Automatically designed machine vision system for the localization of CCA transverse section in ultrasound images. Computer Methods and Programs in Biomedicine 109(1), 92-103.

[14] Islam, A., Reza, S. M. S., Iftekharuddin, K. M. (2013). Multifractal texture estimation for detection and segmentation of brain tumors. IEEE Trans Biomed Eng. 60 (11), 3204-3215.

[15] Zhao, L., Wu, W., Corso, J. J. (2013). Semi-automatic brain tumor segmentation by constrained MRFS using structural trajectories. In: Medical Image Computing and Computer-Assisted Intervention MICCAI 2013, Lecture Notes in Computer Science Volume, Vol. 8151. pp. 567-575.

[16] Capelle, A.-S., Colot, O., Fernandez-Maloigne, C. (2004). Evidential segmentation scheme of multi-echo MR images for the detection of brain tumors using neighborhood information. Information Fusion 5(3), 203-216. 
[17] Prastawa, M., Bullitt, E., Moon, N., Van Leemput, K., Gerig, G. (2003). Automatic brain tumor segmentation by subject specific modification of atlas priors. Academic Radiology 10 (12), 1341-1348.

[18] Otsu, N. (1979). A Threshold selection method from gray-level histograms. IEEE Transactions on Systems, Man and Cybernetics 9(1), 62-66.

[19] Dvorak, P., Kropatsch, W. G., Bartusek, K. (2013). Automatic brain tumor detection in T2- weighted magnetic resonance images. Measurement Science Review 13(5), 223-230.

[20] Uher, V., Burget, R., Masek, J., Dutta, M. (2013). 3D brain tissue selection and segmentation from MRI. In: Telecommunications and Signal Processing (TSP), 2013 36th International Conference on. pp. 839-842.

[21] Ruppert, G. C. S., Teverovskiy, L., Yu, C., Falcao, A. X., Liu, Y. (2011). A new symmetry-based method for midsagittal plane extraction in neuroimages. In: International Symposium on Biomedical Imaging: From Macro to Nano.

[22] Bhattacharyya, A. (1943). On a measure of divergence between two statistical populations defined by their probability distribution. Bulletin of the Calcutta Mathematical Society 35, 99-110.

[23] Kropatsch, W. G., Haxhimusa, Y., Ion, A. (2007). Mul- tiresolution image segmentations in graph pyramids. Applied Graph Theory in Computer Vision and Pattern Recognition Studies in Computational Intelligence. 52, 3-41.

[24] Dvorak, P., Bartusek, K., Kropatsch, W. G. (2013). Automated segmentation of brain tumor edema in FLAIR MRI using symmetry and thresholding. In: Progress in Electromagnetics Research Symposium. pp. 936-939.

[25] Cocosco, C. A., Kollokian, V., Kwan, R. K.-S., Pike, G. B., Evans, A. C. (1997). Brainweb: Online interface to a $3 \mathrm{D}$ MRI simulated brain database. NeuroImage 5, 425.

[26] Prastawa, M., Bullitt, E., Gerig, G. (2009). Simulation of brain tumors in MR images for evaluation of segmentation efficacy. Medical Image Analysis 13(2), 297-311.

[27] Dice, L. R. (1945). Measures of the amount of ecologic association between species. Ecology 26(3), 297-302.

[28] Menze, B., Jakab, A., Bauer, S. et al. (2014). The multimodal brain tumor image segmentation benchmark (BRATS). IEEE Transactions on Medical Imaging, Institute of Electrical and Electronics Engineers (IEEE), pp. 33. http://hal.inria.fr/hal-00935640.

Received May 31, 2014. Accepted October 31, 2014. 